\title{
Raumplanung und Fremdenverkehr im Kanton Graubünden
}

\section{Einleitung}

Die augenscheinlichsten Zusammenhänge zwischen Raumplanung und Fremdenverkehr bestehen in den Bereichen Landschaft, Besiedlung und Verkehr. Eine naturnahe und von der Landwirtschaft gepflegte Landschaft, die Größe, Struktur und das Aussehen der Siedlungen sowie die Erreichbarkeit des Fremdenverkehrsortes mit den Verkehrsmitteln bestimmen die Attraktivität einer Fremdenverkehrsregion. Jede Fremdenverkehrsentwicklung ist jedoch mit Eingriffen und Beeinträchtigungen von Landschaft und Besiedlung verbunden. Aufgrund dieser Tatsache stellt sich bei jeder touristischen Entwicklung die Frage des Maßes. Die Raumplanung bietet für die Beurteilung und Lösung dieser Grundsatzfrage eine echte Hilfe, da sie sich mit der Nutzung, Gestaltung und Erhaltung unseres Lebensraumes als zentrales Anliegen auseinandersetzt.

Seit rund zehn Jahren beschäftigen sich Kanton, Regionen und Gemeinden intensiv mit der Raumplanung. Die Raumplanungsarbeiten haben einen beachtlichen Stand erreicht. Es geht nun darum, im Sinne einer Zwischenbilanz die Auswirkungen des bisher erreichten Planungsstandes auf die weitere Fremdenverkehrsentwicklung zu prüfen.

\section{Der Stand der Raumplanung im Kanton Graubünden und seine Auswirkungen auf die weitere Fremden- verkehrsentwicklung}

Im Kanton Graubünden ist seit 1973 das kantonale Raumplanungsgesetz in Kraft. Das Gesetz verpflichtet den Kanton und die Gemeinden zur Raumplanung im gegenseitigen Einvernehmen. Die Gemeinden sind im Kanton Graubünden für die Nutzung, Erschließung und Gestaltung des Gemeindegebietes verantwortlich. Heute verfügen alle Gemeinden über eine Ortsplanung.

\section{Ortsplanungen}

Die Auswertung aller Ortsplanungen zeigt folgende Charakteristiken: a) Die Bauzonen haben eine Fläche von ca. 8000 ha, davon sind schätzungsweise $1 / 2$ bis $2 / 3$ überbaut. Das Fassungsvermögen der Bauzonen beträgt ca. 600000 Einwohner, Gastbetten und Arbeitsplätze (bei einem Stand von rund 360000 ). Die Annahmen für die Einwohner- und Gastbettenentwicklung gemäß den regionalen Entwicklungskonzepten betragen für $\mathrm{Z} 1$ (ca. 1990) 60000 Einwohner und Gastbetten. Demzufolge wären gesamtkantonal betrachtet die Bauzonen drei- bis viermal zu groß.

Die Verteilung der zu großen Bauzonen schwankt regional beträchtlich. Mit wenigen Ausnahmen verfügen die großen Fremdenverkehrszentren und -orte über sehr große Bauzonen: Oberengadin, Flims/Laax, Obersaxen, Lugnez, Klosters, Savognin u. a. - Negative Auswirkungen zu großer Bauzonen sind: Zersiedlung innerhalb der Bauzonen, was später eine Rückzonung aufgrund des Erschließungsstandes verhindert, ungeordnetes und rasches Wachstum der Siedlungen mit den entsprechenden Wirkungen auf den Gemeindehaushalt sowie die kulturelle und soziale Situation der Dorfgemeinschaft, Verminderung der Wirkung der Nutzungsplanung als Steuerungsmittel für die bauliche Entwicklung. Die großen Zentren Davos/Klosters, Oberengadin, Flims/Laax und Lenzerheide erreichen heute eine Schwelle der Trag- und Ausbaufähigkeit (Gleichgewicht zwischen Erholungsraum, Größe der Siedlung und Infrastrukturanlagen). In diesen Fremdenverkehrszentren hat die Reduktion der Bauzonen und die konsequente Ressourcenbewirtschaftung absolute Priorität.

b) Die Entwicklung des Siedlungsbildes in den großen Fremdenverkehrszentren und ihren "Vorortsgemeinden» (z. B. Falera, Lantsch/Lenz) sowie den aufstrebenden «jungen» Fremdenverkehrsorten (Disentis, Breil/Brigels, Obersaxen, Pany/Luzein, S. Bernardino) gibt Anlaß, an der Wirksamkeit der raumplanerischen Maßnahmen zu zweifeln. Eine bedeutende Ursache dieser "geplanten Zersiedlung» ist die zu large Anwendung der Bau- und Gestaltungsvorschriften und die ungenügende und unzweckmäßige Erschließungsetappierung.

Joseph Sauter, Raumplaner BSP/Geograph, Chur 
c) Die Skigebiete, das heißt die erschlossenen und für die künftige Erschließung vorgesehenen Gebiete, umfassen ca. 43000 ha. Davon sind ca. 22000 ha bereits erschlossen. Einzelne Skigebiete erreichen bereits heute einen hohen Sättigungsgrad: Lenzerheide, Oberengadin, Arosa, Parsenn. Über große Reserveflächen (das heißt Erweiterungsmöglichkeiten und Verdichtung innerhalb der groberschlossenen $\mathrm{Ge}$ biete) an Skigebieten verfügen:

\section{Skigebiet}

\section{Obersaxen/Lugnez}

Flims/Laax

Parsenn/Fondei/Duranna

Schamserberg (neues Skigebiet)

Savognin

Scuol/Sent/Ftan

Samnaun

Reservefläche

Die Entwicklungstendenzen beim Ausbau der touristischen Transportanlagen sind: Erhöhung des Transportangebotes durch technische Verbesserungen und Mehrfachanlagen sowie Zusammenschlüsse von bereits erschlossenen Skigebieten (zum Beispiel Lenzerheide-Arosa-Tschiertschen; Stätzerhorn-Brambrüesch-Feldis). Die Erhöhung des Transportangebotes unter dem Motto "Abbau der Warteschlangen» führt eventuell zu einem neuen Entwicklungsschub. Dieser wäre verbunden mit der Ausdehnung des Parkraumes bei den Talstationen, einer weiteren starken Zunahme der Verkehrsbewegungen in Spitzenzeiten und der Erhöhung des Bettenangebotes zur besseren Auslastung der Bahnen. Diese Tendenz zur Verdichtung würde sich in den großen Fremdenverkehrszentren höchst problematisch auswirken.

d) Das Raumdispositiv, definiert als Summe der kommunalen Nutzungsplanungen und der Vorstellungen der regionalen Entwicklungs- und Raumordnungskonzepte sowie kantonaler Sachplanungen, ergibt aus kantonaler Sicht eine regional ausgeglichene und recht zweckmäßige Ordnung. Das Raumdispositiv wurde nach folgenden Grundsätzen und allgemein anerkannten Zielvorstellungen beurteilt:

- Erhaltung der gutgeeigneten landwirtschaftlichen Flächen. In verschiedenen Gemeinden stellen sich Nutzungskonflikte zwischen zu großen Bauzonen und landwirtschaftlichem Kulturland. Die Flächensicherung für die Landwirtschaft ist auch für den Fremdenverkehr von entscheidender Bedeutung.

- Schutz der Landschaften von nationaler und regionaler Bedeutung. Das bis heute erreichte Resultat ist dank einem bereits im Jahr 1970 erstellten kantonalen Inventar (entscheidender Beitrag zur Bewußtseinsförderung) und den provisorischen Schutzmaßnahmen BMR sowie deren definitiven Übernahme in den Zonenplan gut.
- Gleichgewicht Extensiverholungsraum (Ruhegebiet) und Intensiverholungsraum (Skigebiet). Als Grundsatz gilt: In jeder Fremdenverkehrsregion sind dem Skigebiet auch genügend große Ruhegebiete zuzuordnen. Mit Ausnahme des Raumes Lenzerheide und im Prättigau entspricht das räumliche Dispositiv diesem Grundsatz.

\section{Regionale Entwicklungskonzepte}

Mit Ausnahme der Region Bündner Rheintal, Davos und Oberengadin sind alle Regionen förderungswürdig gemäß Investitionshilfegesetz. Die Entwicklungskonzepte sind alle erstellt und genehmigt. Die bedeutendste Wirkung dieser Konzepte ist die Förderung des regionalen Denkens und der regionalen Zusammenarbeit. Die Regionalorganisationen waren jedoch nicht in der Lage, die Planungstätigkeit auf Gemeindeebene entscheidend zu beeinflussen. Die aus raumplanerischer Sicht bedeutendste Aufgabe, sachgerechte Standorte und Lösungen für überkommunale Infrastrukturanlagen $\mathrm{zu}$ finden, $\mathrm{kam}$ in vielen Fällen nicht befriedigend zum Tragen. Die Maßnahmenkataloge der Entwicklungskonzepte, insbesondere in bezug auf Sportanlagen, belegen diese Tatsache eindrücklich. Aus fremdenverkehrspolitischer Sicht muß die Schaffung eines Ausgleichsfonds innerhalb der Region zur Erreichung eines ausgewogenen regionalen Nutzungskonzeptes (zum Beispiel zweckmäßige Zuordnung der intensiv und extensiv genutzten Erholungsräume) und zur Erhaltung unterschiedlich strukturierter Fremdenverkehrsorte (zum Beispiel Fremdenverkehrszentren mit entsprechendem touristischem Angebot und kleine Fremdenverkehrsorte mit einem speziell gearteten Angebot für den ländlichen Tourismus) mit größeren Anstrengungen verfolgt werden.

\section{Kantonale Richtplanung}

Die Vorbereitung und Zurverfügungstellung von Grundlagen für die Planungstätigkeit in Gemeinden und Regionen hat einen bedeutenden Einfluß, so zum Beispiel das kantonale Landschaftsinventar, die Ausscheidung der gutgeeigneten Landwirtschaftsgebiete, die Erstellung des touristischen Eignungskatasters. Die kantonale Sportstättenplanung enthält ein Standortdispositiv, das der Subventionsbehörde des Kantons als Richtlinie und den Regionen als Rahmen für ihre eigenen Überlegungen dient.

Den stärksten Einfluß auf die räumliche Entwicklung übt der Kanton mit der Planung und dem Ausbau des Verkehrsnetzes, insbesondere Straßenbau, aus. Die Regierung sorgt für ein regional ausgeglichenes Investitionsvolumen. Dadurch sind jedoch nur innerhalb der Regionen Prioritätensetzungen möglich. Im wesentlichen folgt die Verkehrspolitik dem Trend der 
Siedlungsentwicklung. Im Rahmen der kantonalen Richtplanung bildet der Verkehr aufgrund seiner Bedeutung für die räumliche Entwicklung sowie der Konflikte mit Landschaft und Siedlung eines der Schwergewichte. Dabei wurden die Grundsätze, Ziele und Maßnahmen der künftigen Verkehrspolitik festgelegt.

\section{Schlußfolgerungen}

Die Größe und Verteilung der Bauzonen und Skigebiete aufgrund der kommunalen Nutzungsplanungen fördert den bisherigen Entwicklungstrend. Das Raumdispositiv als Summe der Nutzungsplanungen und überörtlichen Planungen bildet aber rein qualitativ betrachtet eine brauchbare Grundlage für eine regional ausgeglichene Entwicklung.

\section{Die künftigen Schwerpunkte der Raumplanung}

\section{Grundsätzliches}

Verschiedene Kreise verlangen vermehrte Interventionen des Kantons, um die zu großen Bauzonen und Skigebiete zu reduzieren. Andere Kreise fordern weitere Konzepte, Leitbilder und griffigere Planungsmittel. - Auf der andern Seite zeigt es sich, daß die Anwendung der vorhandenen Planungsmittel und Gesetze die Gemeinden und Bürger bereits heute überfordert, und die Entwicklung der letzten zehn Jahre zeigt, daß mehr Planungsmittel nicht zu einer wirksameren Steuerung der räumlichen Entwicklung geführt haben. Welche Wege sind aufgrund dieser Einsicht in Zukunft einzuschlagen?

a) Die Aufgabenteilung zwischen Kanton und Gemeinden ist aus föderalistischen Gründen beizubehalten. Verbesserungsbedürftig ist hingegen die Koordination zwischen den verschiedenen Planungsebenen und unter den Gemeinden in den Regionalorganisationen.

b) Voraussetzung für die Koordination sind im gegenseitigen Einvernehmen festgelegte Zielvorstellungen über die künftige räumliche Entwicklung. Dies müssen nicht allseitig abgestimmte, widerspruchsfreie kantonale Leitbilder und Konzepte sein. Auch eine einfache Übersicht über die Zusammenhänge zwischen, den räumlichen Entwicklungstendenzen, dem Stand der Planung und den daraus sich ergebenden wichtigsten Zielen und Maßnahmen für die Raumplanung, wie dies der Kanton Graubünden im Rahmen der kantonalen Richtplanung erarbeitet hat, kann genügen. Eine solche Übersicht dient dem Bund, der kantonalen Verwaltung, den Regionalorganisationen sowie den Gemeinden als Orientierungshilfe oder Wegleitung für ihre Planung und Entscheide. Dadurch wird gewährleistet, daß die verschiedenen Maßnah- men rechtzeitig und gezielt aufeinander abgestimmt und im Dienste der anzustrebenden räumlichen Entwicklung eingesetzt werden.

c) Die bedeutendsten raumrelevanten Ziele für den Kanton Graubünden im Bereich des Fremdenverkehrs sind:

- Beschränkung der quantitativen Entwicklung in den großen Fremdenverkehrszentren und -regionen;

- Förderung eines einfachen Tourismus in den «Entleerungsgebieten» (tourisme rural);

- Erhaltung oder Schaffung eines Gleichgewichtes zwischen "Größe der Bauzone» - «Skigebieten» «Ruhegebieten» in jeder Fremdenverkehrsregion;

- Verbesserung der Auslastung der Parahotelbetten und vermehrte Nutzbarmachung der leerstehenden Bausubstanz innerhalb der Bauzonen;

- Förderung der Hotellerie;

- Förderung des Ganzjahrestourismus zur Verbesserung der gleichmäßigen Auslastung der Infrastrukturanlagen;

- Förderung des öffentlichen Verkehrs in den großen Fremdenverkehrszentren bzw. Gewährleistung eines minimalen Angebotes an öffentlichem Verkehr in den «Entleerungsgebieten»;

- Zurückhaltung beim Ausbau der Straßenachsen zu den großen Fremdenverkehrszentren;

- Förderung des Verbindungsstraßenbaus in den abgelegenen Gebieten («Entleerungsgebieten»);

- Entlastung der stark belasteten Dörfer vom Durchgangsverkehr;

- größte Zurückhaltung bei der Erschließung neuer Gebiete mit touristischen Transportanlagen bzw. differenzierte Bewilligungserteilung für touristische Transportanlagen je nach Stand der touristischen Entwicklung.

\section{Aufgaben von Gemeinden, Regionen und Kanton}

Zur Erreichung der Ziele sind Maßnahmen auf verschiedenen Ebenen notwendig. Dem kantonalen Wirkungsfeld sind folgende Aufgaben zuzuordnen:

a) Verbesserung der Koordination und Information: Durch rechtzeitige Koordination zwischen den verschiedenen Planungsträgern und Planungsebenen sowie innerhalb der Verwaltung können negative Auswirkungen von Bauten und Anlagen verhindert und die positiven Auswirkungen verstärkt werden. Die Koordination umfaßt gegenseitige Information sowie offene und schrittweise Austragung der Konflikte. Die Anwendung der vorhandenen Planungsmittel auf Gemeindeebene kann nur durch eine intensivierte Beratungstätigkeit verbessert werden.

b) Einflußnahme auf die räumliche Entwicklung durch Verkehrsmaßnahmen: Der öffentliche Verkehr soll durch folgende Maßnahmen gefördert werden: Ver- 
knüpfung zwischen der RhB und der Reisepost in den Regionszentren, Einführung von Skibussen in den Fremdenverkehrsregionen, Verminderung der Konkurrenzierung zwischen Bahn und Straße in den Talachsen durch Zurückhaltung beim Straßenausbau. Auf die Gestaltung des Parkplatzangebotes bei den Talstationen der touristischen Transportanlagen soll über die Straßengesetzgebung vermehrt Einfluß genommen werden. Auf kommunaler Ebene sind in den großen Fremdenverkehrsorten Verkehrsberuhigungsmaßnahmen erforderlich.

c) Differenzierte Konzessionierungspolitik für Luftseilbahnen und Skilifte: Der Kanton kann aufgrund der bestehenden Gesetzgebung eine differenzierte Konzessionierungspolitik betreiben. Diese sollte darin bestehen, daß bei Neuerschließungen größte Zurückhaltung geübt wird und in den großen Fremdenverkehrszentren Bewilligungen und Konzessionen restriktiv erteilt werden.

d) Aufbau einer konstanten Genehmigungspraxis: Das Bundesgesetz über die Raumplanung bildet eine gute Grundlage für die Prüfung der Ortsplanungsrevision. Es ist wichtig, daß in Zukunft diesen Grundsätzen des Gesetzes im Rahmen des Genehmigungsverfahrens Nachachtung verschafft wird. Eine konstante Genehmigungspraxis unter Berücksichtigung der Grundsätze des Raumplanungsgesetzes ist für die qualitative Verbesserung der Ortsplanungen entscheidende Voraussetzung.

e) Laufende Raumbeobachtung: Die räumliche Entwicklung im Kanton soll mit einfachen Mitteln laufend beobachtet werden. Die Ergebnisse dieser Beobachtungen bilden Grundlagen für die Überprüfung der Raumordnungspolitik sowie die Sensibilisierung der Bevölkerung für raumplanerische Probleme.

Die Gemeinden sind hauptverantwortlich für die Anpassung und Verbesserung der Nutzungsplanung. Aus der Sicht des Fremdenverkehrs sind dabei folgende Schwerpunkte zu setzen:

a) Ordnung im Landschaftsraum: In Gemeinden, in denen für die Landschaften von nationaler, kantonaler und regionaler Bedeutung noch keine Schutzzonen ausgeschieden wurden, sind entsprechende Maßnahmen zu treffen. In Gebieten mit intensiver touristi- scher Nutzung sind entsprechende Ausgleichsräume (Ruhegebiete) auszuscheiden. Die restriktive Behandlung von Bauten außerhalb der Bauzonen gemäß dem Bundesgesetz über die Raumplanung ist weiterzuführen. Durch vermehrte Informationen ist bei der Bevölkerung das Verständnis für diese restriktiven Maßnahmen zu fördern.

b) Erhaltung der Landwirtschaft: Die Landwirtschaft wird durch die touristische Entwicklung sehr stark bedrängt, so durch Ausdehnung der Siedlungen auf dem gut geeigneten, betriebsnahen Kulturland, durch Einengung der Betriebe und die Schwierigkeit, am Dorfrand geeignete Standorte für neue Betriebe zu finden. Um der Landwirtschaft die gut geeigneten und betriebsnotwendigen Flächen in Siedlungsnähe oder innerhalb der Siedlungen zu erhalten, sind Auszonungen vorzunehmen, Nutzungskonzentrationen, Etappierungen und Umlegungen durchzuführen.

c) Verkleinerung zu großer Bauzonen: In vielen Fremdenverkehrsgemeinden sind die Bauzonen zu gro $B$ bemessen. Auf entsprechende negative Folgen davon ist bereits hingewiesen worden. Die Nutzungsplanung hat ihre Bedeutung als Steuerungsmittel weitgehend verloren. Mit geeigneten Maßnahmen soll versucht werden, bedeutende Nutzungskonflikte zu lösen und die Nutzungsplanung wieder vermehrt als wirksames Steuerungsinstrument für eine geordnete Entwicklung einzusetzen.

d) Strukturförderung mittels spezifischer Nutzungsvorschriften: Die rasante Entwicklung der Zweitwohnungen bedroht die traditionelle Hotellerie. Aufgrund der starken Bodenpreisentwicklung ist es der ansässigen Bevölkerung nicht mehr möglich, Wohnraum zu finanziell vernünftigen Bedingungen zu erstellen. Es soll deshalb im Rahmen der Nutzungsplanung versucht werden, mit spezifischen Nutzungsvorschriften die Erstellung von Zweitwohnungen in bestimmten Zonen zu erschweren bzw. zu verhindern (zum Beispiel Dorfkernzonen), die traditionelle Hotellerie durch entsprechende Vorschriften (zum Beispiel Ausnützungsbonus u.a.) zu fördern und Zonen für den Wohnungsbau von Einheimischen festzulegen. Dabei ist zu beachten, daß die spezifischen Nutzungsvorschriften nicht nur Bezug nehmen auf kurzfristige Entwicklungstendenzen, sondern langfristigen fremdenverkehrspolitischen Überlegungen gerecht werden. 